\title{
artigo
}

\section{Saúde coletiva indígena e análises de depósitos tecnogênicos da comunidade Ikpeng: parque indígena do Xingu}

Indigenous collective health and analysis of technogenic deposits of the Ikpeng community: Xingu indigenous park Salud colectiva indígena y análisis de depósitos tecnogénicos de la comunidad Ikpeng: parque indígena Xingu

\section{RESUMO}

Objetivo: Caracterizar os depósitos tecnogênicos construídos na comunidade indígena Ikpeng, localizada no Parque Indígena do Xingu no nordeste Estado do Mato Grosso. Método: estudo transversal, quantitativo, descritivo e exploratorio realizado por meio de análises laboratoriais do solo em relação aos depósitos existentes quanto a característica, natureza e dimensão. Resultados: Indicam que os dejetos são formados principalmente por resíduos resultantes do estilo de vida da comunidade, destacando-se pilhas e embalagens. Nas análises microbiológicas do solo não foram evidenciadas presença de nematóides (parasitas) prejudiciais a saúde da população, porém, no que se refere a análise da microbiota de vida livre presente na superfície do solo apresentou quantidade siginifivativamente desses organismos que podem levar a doenças infecto-contagiosas. As análises químicas mostraram que os nutrientes: potássio, fósforo e zinco estão acima da média em todas as amostras.Conclusão: A presença depósitos tecnogênicos no solo podem futuramente poluir e fragilizar a saúde da população indígena.
\end{abstract}

DESCRITORES: Gerenciamento de Resíduos; Cultura Indígena; Saúde de Populações Indígenas; Gestão Ambiental.

\section{ABSTRACT}

Objective: To characterize the technogenic deposits built in the Ikpeng indigenous community, located in the Xingu Indigenous Park in the northeastern state of Mato Grosso. Method: cross-sectional, quantitative, descriptive and exploratory study carried out through laboratory analyzes of the soil in relation to the existing deposits in terms of characteristic, nature and dimension. Results: They indicate that the wastes are formed mainly by residues resulting from the lifestyle of the community, especially batteries and packaging. In the microbiological analyzes of the soil, the presence of nematodes (parasites) that were harmful to the population's health was not evidenced. contagious. Chemical analyzes showed that the nutrients: potassium, phosphorus and zinc are above average in all samples.Conclusion: The presence of technogenic deposits in the soil may in the future pollute and weaken the health of the indigenous population.

DESCRIPTORS: Waste Management; Indigenous Culture; Health of Indigenous Populations; Environmental management.

\section{RESUMEN}

Objetivo: Caracterizar los depósitos tecnogénicos construidos en la comunidad indígena Ikpeng, ubicada en el Parque Indígena Xingu en el estado nororiental de Mato Grosso. Método: estudio transversal, cuantitativo, descriptivo y exploratorio realizado mediante análisis de laboratorio del suelo en relación a los depósitos existentes en cuanto a características, naturaleza y dimensión. Resultados: Indican que los desechos están formados principalmente por residuos resultantes del estilo de vida de la comunidad, especialmente baterías y empaques. En los análisis microbiológicos del suelo no se evidenció la presencia de nematodos (parásitos) perjudiciales para la salud de la población. contagioso. Los análisis químicos mostraron que los nutrientes: potasio, fósforo y zinc están por encima del promedio en todas las muestras. Conclusión: La presencia de depósitos tecnogénicos en el suelo puede en el futuro contaminar y debilitar la salud de la población indígena.

DESCRIPTORES: Manejo de Residuos; Cultura indígena; Salud de las poblaciones indígenas; Gestión ambiental.

RECEBIDO EM: 29/01/2021 APROVADO EM: 05/02/2021

\section{Damiane Santos Cerqueira}

Mestre em Ciências Ambientais e Saúde pela Pontifícia Universidade Católica de Goiás, Goiânia, Brasil. Enfermeiro de Campo na área assistencial de atenção primária junto as populações indígenas no Parque Îndigena do Xingu.

ORCID: 0000-0002-3162-6159 


\section{Julio Cezar Rubin de Rubin}

Doutor em Geociências pela UNESP (Rio Claro). Professor na Pontifícia Universidade Católica de Goiás, Goiânia, Brasil. ORCID: 0000-0001-9789-2559

\section{Marieli Basso Bolpato}

Enfermeira e Fisioterapeuta. Doutora em Saúde Pública pela Universidad Americana; Doutora em Psicologia pela Pontifícia Universidade Católica de Goiás, Goiânia, Brasil. Professora na Universidade Federal de Mato Grosso, MT, Brasil.

ORCID: 0000-0002-7923-2343

\section{Jefferson Carlos de Oliveira}

Enfermeiro, Mestre em Enfermagem pelo Centro Universitário São Camilo, Docente do Curso de Enfermagem e Pós-Graduação em Urgência e Emergência - Centro Universitário Anhanguera de São Paulo - Vila Mariana, São Paulo - SP.

ORCID: 0000-0002-5258-7099

\section{INTRODUÇÃO}

D e acordo com o Instituto Brasileiro de Geografia e Estatistica (IBGE) no Brasil existem 305 etnias indígenas e 274 línguas, revelando elevada diversidade cultural, além de quadros epidemiológicos e demográficos bastante distintos ${ }^{1}$. Os indígenas possivelmente atravessam um complexo processo de transição epidemiológica, no qual, ainda que as doenças infecciosas e parasitárias persistam como importante causa de óbito, percebe-se, paralelamente, um aumento expressivo de doenças crônicas não transmissíveis e causas externas relacionadas à ocorrência de óbitos ${ }^{2}$.

Desde os primeiros contatos entre os indígenas e a sociedade envolvente, é considerável o número de doenças levadas aos índios pelos colonizadores, muitas delas com efeitos fatais para a sociedade indígena. Cada grupo que vive em situação de isolamento possui uma combinação peculiar de agentes com os quais convive, seus efeitos fatais parecem atenuar-se por força desta convivência ${ }^{3}$.

Quando as populações indígenas são expostas a outros germes, vírus ou parasitas presentes no solo, a mortalidade é significativamente alta. Deste modo, a condição social de vida é um determinante importante quando se refere à saúde de uma população, principalmente quando se trata do público vulnerável as condições de saúde e à precariedade da infraestrutura básica nos domicílios ${ }^{4}$.

Neste contexto vale ressaltar que os depósitos tecnogênicos são aqueles ori- ginados pela ação humana, podendo ser classificados com construídos, induzidos e modificados ${ }^{9}$, e para outros autores são considerados como paisagens modificadas pela ação do ser humano, com influência direta na dinâmica natural da formação dos processos naturais da região podendo ser responsáveis por causar a entrada de substâncias tóxicas nos ambientes 5 .

Diante do exposto é possível notar a importância da atenção a saúde indígena, uma vez que, que os povos indígenas representam $0,4 \%$ da população nacional ${ }^{5}$, apresentando indicadores de saúde duas a três vezes piores quando comparados aqueles da sociedade brasileira, com altas taxas de doenças endêmicas, carência de assistência médica e doenças crônicas ${ }^{6}$.

Neste sentido o Sistema Único de Saúde (SUS) criou um subsistema coordenado pelos Distritos Sanitários Especiais Indígenas (DSEI), que executam as ações de saúde nos territórios indígenas voltado a atençao a saúde indígena local realizada sendo formado por uma rede de serviços estruturados no interior das terras indígenas, e é responsável pelo provimento de ações e serviços de atenção básica à saúde para a população indígena, baseando-se no modelo de vigilância à saúde ${ }^{7}$. A continuidade da assistência especializada é realizada em outros níveis de atenção dentro do SUS nos municípios referências ${ }^{8}$.

$\mathrm{O}$ presente estudo enfatizou a comunidade indigena dos Ikpeng pertencente à família linguística Karib que vivem atualmente em quatro aldeias: Moygu, Arayo, Tupara e Rawo alicerçados em um posto indígena localizado no Polo Base Pavuru, no
Parque Indígena do Xingu. Sua população foi estimada em 347 indígenas, de acordo com o Sistema Local de Informação.

Em suma, a pesquisa justificou-se por abordar os depósitos tecnogênicos construídos na comunidade Ikpeng no Parque Indígena do Xingu, os quais podem alterar as características do meio ambiente (solo) e afetar a saúde da comunidade.

Por conseguinte, o presente estudo objetivou-se em caracterizar os depósitos tecnogênicos construídos na comunidade indígena Ikpeng e estabelecer por meio de análises laboratoriais os aspectos ambientais do solo e as possíveis implicações a saúde da população.

\section{MÉTODO}

Trata-se de uma pesquisa de campo, de abordagem quantitativa, descritiva e exploratória, com atividades de campo, gabinete e laboratório. $\mathrm{Na}$ pesquisa de campo foi percorrido da área de pesquisa para identificação, caracterização preliminar e quantificação dos depósitos tecnogênicos. $\mathrm{Na}$ caracterização foram identificados os constituintes, comprimento e largura dos depósitos utilizando uma trena de 30 metros.

Foram selecionados 5 (cinco) pontos para a caracterização dos depósitos tecnogênicos (DT) e coleta de amostra de solos, sendo assim denominados: DT 1 e 2 - casa de Napiki Ikpeng (Bebeto); DT 3 - casa de Mogori Ikpeng; DT 4 - casa Nugare Ikpeng (Tugu); DT 5 - local com grande quantitativo de descarte de pilhas.

A comunidade indígena Ikpeng, vive na aldeia Moygu pertencente ao Polo Base 
Pavuru é constituída por 22 casas, com população de 347 indígenas. A presença de depósitos tecnogênicos na comunidade se tornou um fator preocupante, uma vez que podem contaminar o solo, o lençol freático e tornar-se locais propícios para o abrigo de vetores transmissores de doenças como dengue, malária, parasitoses, diarreias, leishmaniose dentre outras.

A quantificação e identificação dos constituintes foi realizada por estimativa e a obtenção das coordenadas geográficas com o Global Positioning System (GPS) dos tipos Garmim e Etrex H. Posteriormente foram realizadas análises dos solos coletados e encaminhadas aos laboratório específicos (Laboratório JEM Análise Agrícola (Aparecida de Goiânia/GO) para análise microbiológica com o intuito de identificar a presença de fungos e nematoides e, para o Laboratório Solocria Agropecuário Ltda (Goiânia/GO), para análise química (cádmio, cromo, níquel e chumbo).

$\mathrm{Na}$ coleta de amostras de solos utilizou-se os seguintes procedimentos: com uma cavadeira tipo boca de lobo foram obtidas amostras nas profundidades de 0,0-0,20 metros e 0,20-040 metros, acondicionamento em sacos de plástico, lacradas e etiquetadas.

Em relação ao gabinete, foram realizadas pesquisas bibliográficas; análise de todos os depósitos tecnogênicos construídos e identificados inicialmente; escolha dos depósitos a serem detalhados, quantificação, caracterização dos rejeitos constituintes; construção de tabelas demonstrativas. Posteriormente as amostras de solos foram encaminhadas para análise laboratoriais e químicas. Foram encaminhadas 15 amostras de cinco depósitos tecnogênicos na identificação de fatores químicos (chumbo, cromo, niquel entre outros) e fatores microbiológicos (bactérias e fungos).

A coleta deu-se no iniciou em outubro de 2018 por meio de observação por parte do pesquisador que atua como enfermeiro junto a essa população há sete anos. As amostras de solo foram coletadas em maio de 2019. A identificação e caracterização de uma anomalia geoquímica em um solo só são possíveis por meio de análises de laboratório, onde as amostras de solo são analisadas e caracterizadas de acordo com a sua composição.

Já, no caso da análise da microbiota de vida livre e com isolamento para a análise das espécies

Fusarium spp.,

Rhizoctonia spp. e

Trichoderma spp.

foram evidenciados

nas amostragens

diferentes

níveis desses

microrganismos

o que mostrou-se

preoupante para

o surgimento de

doenças infecto-

contagiosas.

Os critérios de inclusão foram depósitos tecnogênicos que apresentassem diversidade de material (dejetos) e que estivessem em um raio de até 50 metros das moradias. Já nos critérios de exclusão foram descartados depósitos tecnogenicos com pouca representatividade de cultura material, com excessão de acúmulo de pilhas, que tem uma significância muito alta em termos de elemento químico presente nesse local.

$\mathrm{O}$ projeto de pesquisa foi aprovado no Comitê de Ética de Pesquisa da PUG Goiás sobre o CAAE: 12738619.6.0000.0037, respeitando a Resolução no 466, de 12 de dezembro de 2012 que dispõe de diretrizes e normas regulamentadoras de pesquisas envolvendo seres humanos?. Porém, vale ressaltar que a pesquisa não envolveu diretamente pessoas e, a possibilidade de riscos existentes relacionou-se apenas ao pesquisador durante o período das coletas de dados.

\section{RESULTADOS}

No decorrer das visitas no entorno das residências domiciliares da comunidade, percebeu-se uma grande quantidade de resíduos expostos nos diversos espaços da aldeia, como roupas velhas, chinelos, latas, garrafas de refrigerante, latas de óleo, fraldas descartáveis, carcaças de aparelhos de televisão, fogões velhos, bacias de plástico e alumínio, pilhas, frascos de óleo usados para abastecimento de barcos, entre outros. Verificou-se também a existência de alguns buracos ou valas que provavelmente são utilizados para a queima de alguns determinados tipos de resíduos produzidos pela comunidade.

$\mathrm{Na}$ Tabela 1 em relação as análises microbiológicas laboratoriais das amostras coletadas do solo, não foi detectada a presença de nematóides (parasitas). Já, no caso da análise da microbiota de vida livre e com isolamento para a análise das espécies Fusarium spp., Rhizoctonia spp. e Trichoderma spp. foram evidenciados nas amostragens diferentes níveis desses microrganismos o que mostrou-se preoupante para o surgimento de doenças infecto-contagiosas.

O solo do DT 1 apresentou a maior quantidade desses organismos $(972-10$ $\mathrm{cm} ; 1.560-20 \mathrm{~cm} ; 420-30 \mathrm{~cm}$ ). A presença desses fungos, quando em grandes 
quantidades podem comprometer o cultivo de algumas culturas, porém elas têm um papel importante no ecossistema, auxiliando na ciclagem de nutrientes. Ainda no DT1, houve uma grande presença das espécies (Trichoderma sp - 16.000), a concentração desses microrganismos no solo, podendo contribuir com o desequilíbrio natural deste ambiente, bem como, propiciar o surgimento de doenças infecto-contagiosas.

Já em relação ao DT 3 e 4 os mesmo mantiveram equilíbrio natural e aceitavél das das unidades formadoras de colônias no solo. Entrentanto, na análise do DT 5 houve pouca presença de microrganismos de vida livre $(320-10 \mathrm{~cm} ; 192-20 \mathrm{~cm}$; $80-30 \mathrm{~cm}$ ). Esses resultados podem estar associados com a localização do deposito uma vez que se encontra mais distante da aldeia onde a presença dos indígenas acontece com menos frequência quando comparando com os demais locais de estudo.

Conforme Tabela 2 onde foram realizadas análises químicas do solo percebeu-se que os nutrientes potássio $(\mathrm{K})$, fósforo $(\mathrm{P})$ e zinco $(\mathrm{Zn})$ mostraram-se acima da média em todas as amostras, conforme dados expostos pela Embrapa 10. A amostra retirada a $10 \mathrm{~cm}$ de profundidade do DT2 foi a que apresentou os maiores valores de potássio $(176,4)$ e de fósforo $(255,0)$. Com relação ao zinco, foram observados altos valores nas três profundidades de amostra do DT5 $(81,0-10 \mathrm{~cm} ; 48,3-20 \mathrm{~cm} ; 18,9-30$ $\mathrm{cm})$. Embora os valores de zinco estejam acima da média preconizada pela Embrapa10 para fertilidade do solo, não são considerados tóxicos para a saúde humana.

Tabela 1: Análises Microbiológicas em relação as amostras coletadas no solo e a microbiota das espécies fusarium spp., rhizoctonia spp. E trichoderma spp. Goiânia, 2021.

\begin{tabular}{|c|c|c|c|c|c|c|c|}
\hline DT & Profundidade (cm) & Fusarium Solani & Fusarium sp. & Rhizoctonia sp. & Thichoderma sp. & Crinonemella sp. & Vida Livre \\
\hline \multirow{3}{*}{ DT1 } & 10 & 0 & 1.333 & 4 & 16.000 & 0 & 610 \\
\hline & 20 & 0 & 1.000 & 5 & 5.000 & 0 & 70 \\
\hline & 30 & 0 & 1.667 & 4 & 4.667 & 0 & 84 \\
\hline \multirow{3}{*}{ DT2 } & 10 & 1.667 & 7.000 & 9 & 2.667 & 0 & 972 \\
\hline & 20 & 0 & 2.333 & 8 & 1.667 & 0 & 1.560 \\
\hline & 30 & 0 & 3.000 & 3 & 1.333 & 0 & 420 \\
\hline \multirow{3}{*}{ DT3 } & 10 & 1.000 & 3.667 & 0 & 8.000 & 0 & 1.240 \\
\hline & 20 & 0 & 1.667 & 3 & 5.000 & 0 & 1.060 \\
\hline & 30 & 1.333 & 2.667 & 1 & 2.667 & 0 & 710 \\
\hline \multirow{3}{*}{ DT4 } & 10 & 0 & 2.333 & 0 & 3.000 & 10 & 190 \\
\hline & 20 & 0 & 1.333 & 1 & 3.333 & 0 & 370 \\
\hline & 30 & 0 & 1.667 & 2 & 3.333 & 0 & 190 \\
\hline \multirow{3}{*}{ DT5 } & 10 & 0 & 1.667 & 2 & 0 & 0 & 320 \\
\hline & 20 & 0 & 667 & 3 & 0 & 96 & 192 \\
\hline & 30 & 0 & 2.000 & 0 & 0 & 110 & 80 \\
\hline
\end{tabular}

\section{Tabela 2 - Interpretação das análises químicas do solo- Goiânia, 2021.}

\begin{tabular}{|c|c|c|c|c|c|c|c|c|c|c|c|}
\hline Local & Prof & $\mathrm{Ca}$ & $\mathrm{Mg}$ & Sat. Al & $\mathrm{pH}$ & Sat. Bases & $\mathrm{Zn}$ & $\mathrm{Pb}$ & $\mathrm{Cd}$ & $\mathrm{Cr}$ & $\mathrm{Ni}$ \\
\hline & $\mathrm{Cm}$ & cmolc dm-3 & $\%$ & $\mathrm{CaCl} 2$ & $\%$ & mg dm-3 & & & ppm & & \\
\hline DT -1 & 10 & baixo & baixo & baixa & médio & média & alto & baixo & baixo & baixo & baixo \\
\hline DT -1 & 20 & baixo & baixo & muito alta & baixo & baixo & baixo & & & & \\
\hline DT -2 & 20 & baixo & baixo & baixo & médio & médio & alto & & & & \\
\hline DT - 4 & 20 & baixo & baixo & baixo & médio & médio & baixo & baixo & baixo & baixo & baixo \\
\hline DT - 4 & 30 & baixo & baixo & alta & médio & médio & baixo & & & & \\
\hline DT - 5 & 10 & baixo & baixo & muito alta & baixo & baixo & alto & & & & \\
\hline
\end{tabular}




\section{artigo}

\begin{tabular}{|c|c|c|c|c|c|c|}
\hline DT - 5 & 20 & baixo & baixo & muito alta & baixo & baixo \\
\hline DT - 5 & 30 & baixo & baixo & muito alta & baixo & baixo \\
\hline
\end{tabular}

Fonte: Solocria Agropecuário, Goiãnia (2019)

Com relação à fertilidade de solos, os locais dos DT 1 e DT 5 apresentaram solos ácidos, pelos baixos valores de saturação de bases (Sat. Bases < 50\%)10. Também, os altos valores de saturação de alumínio e da combinação hidrogênio e alumínio (Sat $\mathrm{Al} ; \mathrm{H}+\mathrm{Al}$ ) favorecem a pouca fertilidade desses locais.

As amostras de solo coletado no DT3, em profundidade de $30 \mathrm{~cm}$ não teve classificação satisfatória para os resultados das análises químicas, uma vez que as amostras coletadas demonstraram ser insatisfatória na obtenção desses dados. Porém os resultados dessas amostras analisadas possibilitaram averiguar resultados que caracterizam a qualidade de fertilidade do solo revelando ser um solo eutrófico (produtivo), devido as variáveis como saturação de bases maiores de $50 \%$ e os baixos valores de saturação de alumínio (Sat. Al) e da combinação de hidrogênio e alumínio $(\mathrm{H}+\mathrm{A})$.

\section{DISCUSSÃO}

O estudo realizado no território indígena do XINGU na aldeia Moygu, permitiu a identificação de cinco DTs, evidenciados pela presença e acúmulo de resíduos sólidos descartados de forma inadequada no ambiente. As análises químicas e microbiológicas do solo evidenciaram que a curto e longo prazo podem impactar na saúde da população e na qualidade do solo ${ }^{11}$.

Corroborando com os achados autores confirmam que os depósitos tecnogênicos podem ser considerados como locais apropriados para a proliferação de vetores favorecendo doenças como diarréicas (aguda, disenteria, febre tifóide) observadas principalmente em crianças e adultos moradores de áreas com saneamento básico precário ${ }^{6}$.

No tocante, as doenças diarréicas persistem como uma das principais causas de óbito entre as crianças indígenas, antes que completem cinco anos de ida$\mathrm{de}^{9}$. Os vetores que são encontrados nos depósitos tecnogênicos na aldeia não só causam doenças, eles também agem como uma barreira para o desenvolvimento destas aldeias, acarretando problemas com o solo ${ }^{12}$.

Portanto, existem duas maneiras pelas quais as doenças transmitidas por vetores na aldeia: a mecânica ocorre quando um vetor simplesmente carrega microorganismos patogênicos em seu corpo e os transfere para alimentos, que então consumimos $^{14}$. E, pelo desperdício de equipamentos elétricos e eletrônicos, chamado "lixo eletrônico", que contêm substâncias perigosas liberadas ou geradas diretamente após o descarte ou durante o processo de reciclagem ${ }^{2}$.

Neste sentido, os resíduos perigosos, se não forem adequadamente gerenciados, podem causar efeitos adversos à saúde das populações que moram perto dos locais onde são despejados ou processados. A contaminação de diferentes matrizes ambientais, incluindo alimentos, água, solo e ar, representa um risco à saúde dessas populações ${ }^{13}$.

O contexto apresentado acima ressalta os impactos a saúde e ao meio ambiente que podem ser associados aos depósitos tecnogênicos, pois o descarte inadequado prejudica de maneira direta o meio ambiente e a saúde ${ }^{17}$. As populações indígenas estão gerando muitos resíduos e não podem lidar com isso de maneira sustentável, o que é um grave problema e deve ser abordado por políticas de Estado ${ }^{14}$.

Portanto, nesse cenário atual é preciso a integração da equipe multiprofissional que atuam junto à saúde indígena na construção e no planejamento de ações voltadas em conscientizar as comunidades nas aldeias sobre os resíduos que estão sendo gerado e suas possíveis consequências na saúde e no meio ambiente ${ }^{4}$.Neste contexto, faz se importante o fortalecimento das relações entre as instituições não governamentais, gestores, equipe de saúde e comunidades, na rotina de intervenções para manejo adequado dos resíduos ${ }^{15}$.

A quantidade de resíduos coletada no presente estudo revela a necessidade de buscar, com a participação das comunidades indígenas, formas de manejo e controle. Tais práticas de manejo não integravam o cotidiano das comunidades indígenas no passado, pois não ocorria o consumo de produtos industrializados.

Ficou evidente durante esta pesquisa e nas ações educativas e práticas desenvolvidas com a comunidade Ikpeng, a grande quantidade de resíduos sólidos cada vez mais visíveis no entorno das casas, no pátio e nos quintais da aldeia. Este problema requer o planejamento e realização de uma política de logística reversa, bem como planos de cuidados referentes a gestão de resíduos sólidos ${ }^{16}$.

Os resultados dessa pesquisa mostraram a ausência de nematódeos e/ou parasitas no solo e a presença da microbiota de vida livre. Fato esse que corrobora com o estudo onde observaram a interação entre os microrganismos de solos e os nutrientes como sendo importantes para a manutenção da ciclagem desses, mantendo assim a integridade do solo $^{17}$.

$\mathrm{Na}$ área de estudo a presença de metais pesados, como o zinco e o alumínio ainda é baixa, estão abaixo dos padrões de prevenção para a saúde humana, porém por outro lado caracterizam um solo com excesso de nutrientes corroborando com um estudo realizado em um reservatório de abastecimento de água em Pelotas (RS), o qual foi construído sobre depósitos com interferência humana, encontrando altos valores de concentração de metais pesados, entre eles o zinco, o qual é um composto inorgânico resultante da degradação de produtos industriais ${ }^{18}$.

Em suma, é importante ressaltar que deposítos tecnogênicos são um indicativo de que é necessário ter maior cuidado com 
relação à disposição de material tecnológico nos territórios indígenas. Visto que a degradação desse tipo de material é lenta, podendo influenciar na contaminação do solo, e com isso afetar diretamente a saúde da população indígena local ${ }^{19}$.

Diante do expsoto faz-se necessário o controle e gestão dos resíduos sólidos integrado à rotina das aldeias devendo contemplar conhecimentos e o modo de vida dos povos indígenas. A falta de planejamento e de ações estratégicas de gerenciamento do destino final de resíduos sólidos podem acarretar impactos graves na saúde dos povos indígenas e no ambiente em que eles estão inseridos ${ }^{20}$.

\section{CONCLUSÃO}

Concluiu-se que implicações registradas nos cinco depósitos tecnogênicos estudados não causam riscos na qualidade da saúde desta comunidade, porém vale ressaltar que a longo prazo, esses resíduos podem aumentar em proporções de forma à impactar não somente a saúde dessa população, como o meio ambiente que os permeiam.

As análises microbiológicas de amostras de solo, realizadas nestes locais permitiram averiguar que existem algumas espécies de fungos, porém em quantidades que proporcionam o equilíbrio, e contribuem para ciclagem natural do solo, neste sentido, os achados não retratou danos ao solo atualmente.

No contexto atual, faz se importante a potencialização da equipe multiprofissional que atuam junto à saúde indígena, na construção e no planejamento de ações voltadas em conscientizar as comunidades, sobre os resíduos que estão sendo gerados, seus impactos no agravamento da saúde e no meio ambiente.

\section{REFERÊNCIAS}

1. Kabad JF, Pontes ALDM, Monteiro S. Relações entre produção cientifica e políticas públicas: o caso da área da saúde dos povos indígenas no campo da saúde coletiva. Rev. Ciência e Saúde Coletiva. 2020; 16(2): 1653-1666.

2. Cerqueira DS, Sila MA, Bolpato MB. Influência das condições socioeconômicas e ambientais associadas a parasitoses intestinais em população indígena Xavante. Rev. Interdisciplinar. 2016; 14(2):148-153.

3. Brasil. Resolução no 420, de 28 de dezembro de 2009. Dispõe sobre critérios e valores orientadores de qualidade do solo quanto à presença de substâncias químicas e estabelece diretrizes para o gerenciamento ambiental de áreas contaminadas por essas substâncias em decorrência de atividades antrópicas. 2009; 178-186.

4. Buhler HF. Análise espacial de indicadores integrados determinantes da mortalidade por diarreia aguda em crianças menores de um ano em regiões geográficas. Rev. Ciência \& Saúde Coletiva. 2014; 19.(1): 4131-4140.

5. Mendes AM, Leite MS, Langdon EJ, Grisotti M. O desafio da atenção primária na saúde indígena no Brasil. Rev. Panamericana de Salud Pública. 2018; 42(1): 184-195.

6. Pedrana L, Pereira MLG, Torrenté MDO, Mota SEDC. Análise crítica da interculturalidade na Política Nacional de Atenção às Populações Indígenas no Brasil. Rev. Panamericana de Salud Pública. 2018; 34(2): 178-191.

7. Coimbra CEA. Emergence of new diseases in: The Xavànte in transition: health, ecology, and bioanthropology in Central Brazil. Ann Arbor. University of Michigan Press. 2014; 12(3): 243-267.

8. Ferreira TF, Santos AMD, Oliveira BLC, Caldas ADJM. Microbiota produtora de fitase isolada de solo e serapilheira do Bioma Cerrado. Rev Saúde Pública. 2019; 29(3): 1270-1281.

9. Brasil. Ministério da Saúde; Conselho Nacional de Saúde, \& Brasil. Ministério da Saúde; Conselho Nacional de Saúde. (2012). Dispõe sobre diretrizes e normas regulamentadoras de pesquisas envolvendo seres humanos.
10. Embrapa. Manual de métodos de análise de solos. Dados eletrônicos. Editora Embrapa Solos. 2018; 245-281.

11. Fonseca GVR. $O$ direito do índigena de não se integrar na sociedade: funai's responsabilidade civil objetiva da funai. Revista Aporia Jurídica. 2019; 1(10): 77-100.

12. Santiago CD, Leite CMB, Oliveira TMS, Kotaka F. Análise das equipes de pesquisa em saúde e saneamento financiadas pela Funasa. Congresso Nacional da Assemae. 2018; 48(1): 9981007.

13. Maia SF, Rummennig O, Pagliaro H, Rodrigues D, Baruzzi RG. A recuperação populacional dos Txicão (Ikpeng), parque indigena do Xingu, Mato Grosso,Brasil. Revista Saúde dos Povos 2016; 12(1): 101-109.

14. Miyazaki LCP. Depósitos tecnogênicos: uma nova perspectiva de leitura geográfica. Rev.Quaternary and Environmental Geosciences. 2014; 5(2):53-66.

15. Nascimento AD, Chagas AFA, Vasconcelos EA. As divergências em análises microbiológicas para o Ikpeng (Karib). Rev. Brasileira de Línguas Indígenas. 2020;1(2): 19-35.

16. Niemeyer CP. Xamanismo e novas circulações de conhecimento na amazônia indígena. Rev. Políticas Culturais e Povos Indígenas. 2018: 16(1): 122-136.

17. Ferreira AC, Chagas AFA, Barbosa LJG. Termos de parentesco: primeiras reconstruções em Proto-Arara-Ikpeng. Boletim do Museu Paraense Emílio Goeldi. Rev.Ciências Humanas. 2019: 14(1), 101-119.

18. Oreme OI, Sá D. Valorização do cultivo de roças e suas regras para o povo Ikpeng. Cadernos de Agroecologia. 2016; 13(1); 167-188.

19. Ribeiro IM, Lorrayne J, Doula SM.Tradições culturais locais e mudanças climáticas globais. Revista De Extensão E Estudos Rurais. 2018;7(2): 266-272.

20. Ritter EO, Grasel M. Estratégias para a busca da Gestão de Excelência: Um Estudo de Caso no Município de Canarana. Revista de Estudos Sociais. 2016; 18(36), 251-279. 